\title{
Riesgos psicosociales considerados por jueces de familia en decisiones sobre pérdida de patria potestad: Estudio exploratorio*
}

\section{Psychosocial risks considered by family judges in decisions on loss of parental authority: an exploratory study}

\author{
Alejandro Barbosa González**, Rosa Elena Martínez***, \\ Mariano Alexis Piña**** y Carmen Alicia Segura*****
}

\begin{abstract}
*Este trabajo forma parte de un proyecto de investigación financiado por la Universidad Antonio Nariño (UAN) de Bogotá (Colombia).

**Psicólogo y Magister en Psicología Clínica y de la Familia. Docente de la Maestría en Mediación

Familiar (UAN) y Director del Grupo de Investigación Escuela: Sistema Complejo.

Orcid.org/000-0003-1400-2042.E-Mail: abarbosagonzalez@gmail.com

***Psicóloga y Magister en Bioética. Docente en la Maestría en Mediación Familiar de la

Universidad Antonio Nariño (UAN).

****Psicólogo y Magister en Psicología Jurídica y Forense.

*****Psicóloga y Magister en Psicología Clínica y de la Familia. Docente en la Maestría en Mediación Familiar de la Universidad Antonio Nariño (UAN).
\end{abstract}

Los autores agradecen a la Universidad Antonio Nariño (UAN) el apoyo brindado y la financiación del proyecto.

Universidad Antonio Nariño (UAN). Bogotá, Colombia.

\section{Resumen}

La investigación realizada tuvo como objetivo identificar riesgos psicosociales considerados por jueces en decisiones sobre pérdida de la patria potestad. El tipo y diseño de la investigación fue cualitativo. Las fuentes de información eran dos: (1) se preseleccionaron 50 documentos judiciales en las siguientes ciudades de Colombia: Bogotá, Tunja y Duitama y (2) se hizo una entrevista grupal con ocho jueces. Los documentos fueron analizados con un protocolo y para diseñarlo, se determinaron categorías orientadas por los objetivos y sustento teórico. La entrevista consistió en un grupo focal. El audio de la entrevista se transcribió literalmente y se realizó un análisis categorial inductivo. Los hallazgos indican que en la mayor parte de casos, la causal más común corresponde al abandono.
Allí se identifica más un interés por viajar con el niño fuera del país sin tener que pedir permiso al progenitor demandado. Igualmente se observa que poco se tiene en cuenta la interdisciplinariedad y el interés superior del menor dentro de las decisiones. En el momento de tomar la decisión los jueces utilizan más la lógica silogística que la realista. Empero, en la discusión generada dentro del contexto de la entrevista grupal, anteponen la experiencia profesional y posiblemente vivencias propias para describir las situaciones evaluadas (lógica realista). De la misma manera, reconocen riesgos psicosociales que no quedan expuestos en el documento judicial. Finalmente, se encuentra la necesidad de evaluar más detalladamente la historia relacional de la pareja parental, ya que suelen existir secuelas psicológicas que afectan las competencias parentales. 
Palabras clave: Riesgo psicosocial; patria potestad; Jueces de familia; Interés superior del menor.

\begin{abstract}
Suspension of custody as a right of the child may lead to psychosocial risks considered as potential dangers to the well-being of the child, his family or his community in various dimensions. It is therefore important to consider the possibility of these psychosocial risks when making these judicial decisions about the loss of parental rights. The phenomenon of psychosocial risk has been more studied in working environments and also linked to health problems. This fact assigns a new connotation to this study, since it opens the door to the interdisciplinarity from Psychology and Law, when investigating if the psychosocial risks in the judicial decisions on loss of parental authority are taken into account. The research aims to identify psychosocial risks considered by family judges in decisions about loss of custody. The type and design research is qualitative, since one of its general pretensions is the understanding. The sources of information are two. The first corresponds to 50 pre-selected court documents in the Colombian cities of Bogota, Tunja and Duitama. The other is a group interview with 8 family judges. The documents were analyzed by means of a protocol. For the design and implementation of the protocol, categories oriented by interdisciplinary research team objectives, theoretical support and expectations were made. The interview consisted of a focus group interview with family judges (four men and four women). The audio of the interview was transcribed literally and an inductive categorical analysis was made. Results of the analysis of the interview and documents were contrasted. Judicial documents reviewed show that judges use syllogistic logic when seeking to accommodate situations to the causal. Within the judicial decisions it is observed that the best interest of the minor is not privileged. The analysis of the interview in which the use of experience and intuition is observed following a realistic logic, invites us to reflect on aspects such as: the subjectivity of judges in the evaluation, the pressure they face on the number of lawsuits and the speed of judgment, and the need to revise the causal deprivation of liberty superior to one year. The judges manifest a
\end{abstract}

deficit in the interdisciplinary work and the lack of more training in aspects of mental health that would make possible the use of psychological reports and the greater understanding of causes such as disability. It was possible to identify a pattern that leads to the decision of loss of custody power by abandonment (most common causal): (1) Search for legal advice. (2) To promote the notoriety of the physical and emotional distance between the defendant and the child. (3) Establish the claim. (4) Accumulate evidential evidence on the causal. (5) Obtain witnesses. In the discussion generated in the group interview, judges recognize psychosocial risks that are not exposed in the court document or sentence. Finally there is the need to assess in more detail the relational history of the parental couple, as they tend to be psychological consequences that affect parenting skills. The discussion between the researchers of Family Psychology and those of Family Law appear questions that build an interdisciplinary bridge. One limitation of the study was that it was not possible to have sufficient time and resources to conduct group and individual interviews with a greater number of family judges in other cities of the country and achieve more generalizable results. The research question on whether judges take into account the risks to make the decisions is answered that it attends to the grounds provided in the law taking the decision guided by the syllogistic logic. Causals can be equated with psychosocial risks.

Key words: Psychosocial risk; Custody; Family courts; Child's best interests.

\section{Introducción}

Diversos autores e investigadores en el mundo conciben los riesgos psicosociales como situaciones que ponen en peligro el bienestar de una persona, familia o comunidad. La alteración no se refiere exclusivamente al aspecto físico, ya que también se contempla la salud mental y otros eventos asociados con las relaciones familiares y sociales (Barudy \& Dantagnan, 2010; Bueno-Abad, 2005; Moreno, 2001). Por ejemplo Davis y colaboradores (2014) y Pauli-Pott y colaboradores (2017), consi- 
deran como riesgos psicosociales la separación prolongada de los padres, la pobreza y el consumo de sustancias psicoactivas entre otros (SPA). Los riesgos psicosociales se pueden presentar en diferentes ámbitos de las relaciones humanas. Empero, para los efectos prácticos de la investigación realizada, se tomaron en cuenta los citados como así también otros que pudieran surgir en la familia durante el proceso de investigación.

En la actualidad se encuentran estudios sobre riesgos psicosociales en ambientes laborales y también se los relaciona con algunas enfermedades como la obesidad y el trastorno por déficit de atención, pero no investigaciones de riesgos psicosociales y decisiones judiciales de patria potestad. Lo anterior asigna una connotación novedosa a este estudio y abre las puertas para la interdisciplinariedad. Otro aspecto clave y motivo de discusión entre los investigadores de Derecho y Psicología, es el que tiene que ver con el proceso de toma de decisión de los jueces de familia. Allí nace una inquietud en torno a las lógicas en juego al momento de la toma de decisión de pérdida de patria potestad, ya que existen en la legislación colombiana causales tanto objetivas como subjetivas, asunto que otorga al juez un espacio para ser víctima de sus prejuicios o por el contrario acomodar arbitrariamente las situaciones evaluadas a la causal para tomar la determinación. Adicionalmente se encuentra la cantidad de casos en espera de evaluación, lo cual presiona a los jueces de familia para tomar la decisión.

En toda la discusión entre los investigadores de Psicología y Derecho aparecen preguntas que construyen un puente interdisciplinario. Por ejemplo: ¿Los jueces toman en cuenta riesgos psicosociales para tomar las decisiones?, ¿cuáles son las causales más comunes?, ¿las causales existentes en la legislación colombiana se pueden considerar como riesgos psicosociales?, ¿se está realizando un trabajo interdisciplinario que priorice el interés superior del menor?

El método de investigación utilizado es cualitativo. Con relación al tema, Montenegro y Pujol (2009) resaltan que las metodologías se orientan por principios episte- mológicos que se adhieren al origen del conocimiento. En el caso de la investigación cualitativa, una de las pretensiones generales tiene que ver con la comprensión. Al respecto, Bautista (2011) recuerda que en la postura cualitativa se sustituye teleológicamente la explicación, la predicción y el control por la comprensión, el significado y la acción. También el antropólogo Geertz (2003) arguye que la manera más idónea para interpretar y comprender a una persona o su cultura, es a través de la comprensión de los significados, símbolos y conceptos. Lo anterior resalta la importancia de la subjetividad como medio para aproximarse a la comprensión de la realidad.

De la misma manera está la intersubjetividad. Salgado (2007) recuerda que uno de los principios de la investigación cualitativa consiste en que el mundo se encuentra constituido por símbolos y significados. Entonces, la realidad social es intersubjetiva. También Mardones y Ursúa (1987) señalan que lo idóneo para los estudios sociales es la comprensión y no la explicación que propone el positivismo lógico porque el interés de las ciencias sociales y humanas suele centrarse más en la comprensión que en la explicación.

Con relación a los objetivos del estudio realizado, en general los investigadores pretendían identificar si los riesgos psicosociales son tomados en cuenta por los jueces para tomar la decisión de pérdida de patria potestad a alguno de los padres. A nivel más específico, buscaban determinar las causales más comunes y analizar si dichas causales pueden ser consideradas riesgos psicosociales. También explorar si el interés superior del menor es tenido en cuenta a la hora de tomar las decisiones judiciales. Finalmente, revisar si se está realizando un trabajo interdisciplinario en torno a este tipo de temáticas.

\section{La familia y su función}

El concepto de familia cuenta con diversas definiciones. Igualmente, se confi- 
gura y organiza de variadas formas. En términos genéricos, es un grupo social, cultural e histórico que suele pasar por una serie de etapas de desarrollo. En cuanto a las creencias de sus integrantes, tienden a ser conservadoras. En la actualidad no existe otro grupo que funcione mejor con respecto a la socialización y protección del ser humano. Es precisamente el seno de las relaciones familiares, uno de los primeros ámbitos dentro de los cuales la persona comienza a establecer vínculos de afecto, odio y protección. Infortunada y paradójicamente, también aparecen situaciones de riesgo psicosocial. Esto se debe a que no siempre y por diversas razones, la familia no logra cumplir con su función. Según Echeburúa y de Corral (2009), por extraño que parezca, el hogar que suele constituirse en un comienzo en fuente de cariño, protección, cuidado y satisfacción de las necesidades básicas, se puede transformar en un lugar susceptible para la aparición de múltiples problemáticas psicosociales como la violencia o el consumo de SPA. La consecuencia es un deterioro de las competencias parentales, definidas como la capacidad que tienen los progenitores para responder de forma efectiva, flexible y adaptativa frente a las demandas generadas por dificultades vitales, sociales y culturales (Masten \& Curtis, 2000; Waters \& Sroufe, 1983).

También está el concepto de dinámica familiar, el cual corresponde al tipo de interacciones que establecen las personas. Dichas interacciones tienen que ver con los límites, comunicación y jerarquía que se presentan en el diario vivir del grupo (Barrett \& Mcintosh, 1995; Boszormenyi-Nagy \& Spark, 2013; Fuster, 2002; Nardone, Giannotti \& Rocchi, 2003). En el caso de los límites, corresponden a la distancia física y emocional. La comunicación es lo verbal y lo no verbal. Finalmente la jerarquía tiene que ver con la autoridad o liderazgo. La evaluación de los aspectos anteriores permite entender mejor el funcionamiento de la familia.

\section{Patria potestad}

En cuanto a la patria potestad, el artículo 288 del Código Civil Colombiano (2015) expone que la patria potestad tiene que ver con un conjunto de derechos que reconoce la ley a los padres sobre sus hijos no emancipados. Con lo anterior, los padres poseen el derecho para usufructuar, administrar los bienes y representar a los hijos que estén en condición de desventaja y no se encuentren emancipados o sean mayores de edad en interdicción por discapacidad mental absoluta.

La idea fundamental de esta figura jurídica es que por medio de este conjunto de derechos se pueda garantizar a los hijos la protección, bienestar y formación integral desde el momento mismo de su concepción (Código de la Infancia y la Adolescencia, 2006).

Es importante aclarar que los derechos de crianza, educación y corrección de los hijos no se encuentran incluidos allí, ya que están incluidos en lo referente a la custodia y visita (Naranjo, 1996). Diferentes autores hablan más de potestad parental que de patria potestad (Medina, 2008; Montoya, 2010; Parra, 2008; Serrano, 2007). Lo anterior es porque la primera es conceptualmente más amplia y correlativa en términos de derechos y obligaciones. También porque la Constitución Política (1991) y el Código de Infancia y Adolescencia (2006) establecen la importancia de la unidad familiar y el derecho fundamental del niño: a tener una familia y a no ser separado de ella. Esto se relaciona con proporcionarle al niño amor y cuidado personal. Igualmente está el concepto de responsabilidad parental, introducido por el artículo 14 inciso $1^{\circ}$ de la Ley 1098 de 2006. Dicho artículo se considera complementario a la patria potestad. Aquí aparece el deber de propender por la crianza, educación y corrección de los hijos durante el transcurso de su proceso de formación. A pesar de lo expuesto, se continúa utilizando el término patria potestad en la mayoría de los ámbitos. En resumen, la patria potestad busca garantizar que los dere- 
chos del niño se cumplan. Sin embargo, para que el desarrollo del niño se presente en forma adecuada, es importante fijar la atención en la capacidad que tienen los padres para ejercer en forma idónea sus funciones (competencias parentales).

Por el lado de la pérdida de la patria potestad a los padres, las causales previstas en la normatividad civil de Colombia (numeral 2, art. 315, Código Civil) suelen ser caracterizadas como objetivas y subjetivas. Un ejemplo es la discapacidad de la madre o padre y la condena correspondiente a la privación de la libertad superior a un año. Estas dos causales se fijan en la primera denominación, en especial por los medios de prueba a practicar durante el proceso. En algunos casos se considerara suficiente la sentencia que declara la inhabilitación por discapacidad mental o la condenatoria por la comisión de alguna conducta punible que tenga una pena prevista mínima de un año. Para las que se pueden consolidar en la segunda denominación (subjetividad), se encuentran la larga ausencia y el maltrato del hijo o su abandono, ya que pasa a protagonizar un papel relevante el medio de prueba y también el criterio judicial en cada caso en particular. Debido a lo anterior, lo que para un sujeto es abandono o maltrato para otro no.

\section{La subjetividad en las decisiones judiciales}

Siempre existe la posibilidad de que dentro de las decisiones de los jueces aparezca algún matiz de subjetividad. Al respecto, el Juez americano Alex Kozinski (1993) argumenta que los jueces son objeto de una buena dosis de subjetivismo y a menudo irracionalismo. De la misma forma complementa que suelen otorgarle a sus decisiones algo relacionado con lo que les pide el cuerpo en el momento, y posteriormente acuden al arsenal de normas en búsqueda de aquella o aquellas que le permitan confirmar las intuiciones que manejaban desde un primer momento. Finalmente concluye que en todo caso no existen casos difíciles desde dicha premisa, ya que el juez siempre tiene una respuesta, inclusive si tiene que alejarse de la ley y acercarse más a su intuición.

Cuando el juez busca afanosamente acomodar las situaciones a la causal para tomar la decisión, utiliza la lógica silogística. Con relación al tema, Brooks (2002) explicita que dentro de esta lógica se encuentra una subordinada a la ley para la toma de decisiones. Allí se pone en juego un conjunto de operaciones lógico-mecánicas para subsumir el caso a una o unas normas preestablecidas.

Por otro lado se encuentran situaciones en las que el juez utiliza lógicas menos formales para la toma de decisiones. Cuando esto sucede, opera desde una lógica que se denomina realista. Según Garrido, Masip y Herrero (2006), en esta lógica entrarían a jugar algunas preferencias personales en la toma de decisiones. Aquí asuntos como la experiencia con otros casos y posiblemente vivencias propias, podrían entrar a jugar un papel preponderante dentro del proceso de toma de decisión. Como resultado se pueden terminar generalizando las decisiones.

\section{Método}

Un tema fundamental en la investigación cualitativa es la subjetividad. González (2000) la concibe en dos dimensiones, la individual y la social, que convergen durante el transcurso del desarrollo, ya que la subjetividad individual se constituye socialmente, aunque no por determinismos lineales exteriores que transitan de lo social a lo subjetivo, sino en un proceso recíproco en el cual lo social e individual interactúan dinámica y constantemente.

Según Gergen (2007) el campo cualitativo se ha convertido en un importante terreno para la innovación creativa. Los trabajos sobre reflexividad, formas literarias y expresión narrativa, han nutrido este tipo de investigación. Complementa que la mayoría de concepciones se centran en lo individual (el deseo, la identidad, el sufrimiento o historia de vida). Para este destacado pensador 
construccionista social, sería interesante incluir al otro dentro de los estudios para buscar establecer una diferenciación entre el yo $\mathrm{y}$ el otro. Finalmente concluye resaltando que se constituye en un reto importante la utilización de métodos que generen realidades relacionales, en lugar de separaciones y aislamientos. La idea es lograr una conexión integral que se constituiría en un movimiento clave hacía procesos dialógicos y no dirigidos a una plenaria de voces separadas. Según sus palabras: "La meta de la investigación comienza a ser la de incitar el diálogo, que puede sufrir un cambio continuo a medida que se mueve a través de una amplia red" (p. 268).

\section{Técnicas para recolectar la información}

\section{Protocolo.}

Para el análisis de los documentos judiciales se utilizó un protocolo exploratorio diseñado por los investigadores. La intención de la técnica en mención no es la de ostentar validez o confiabilidad como sucede en el caso de instrumentos estandarizados. Sin embargo, se le pidió a un grupo de expertos en temáticas asociadas a la familia (psicólogos jurídicos, de familia y abogados), su revisión y sugerencias. El objetivo consistía en revisar el contenido consignado en las sentencias, ya que el estudio es netamente cualitativo y la cantidad de expedientes con los que se trabajó es muy pequeña para una generalización. El análisis de los expedientes facilitó la planificación de la entrevista grupal con los jueces. Las categorías que se tuvieron en cuenta son las mismas causales y los riesgos psicosociales contemplados en el estudio. En la Figura 1 se presenta una versión abreviada del protocolo utilizado.

\section{Grupo focal (focused group).}

La otra técnica aplicada fue focused group, y se realizó con ocho jueces de familia (cuatro mujeres y cuatro hombres). Su performance se presenta en diferentes ciudades de Colombia (Tunja, Sogamoso, Bucaramanga, Cali y Bogotá).

Los métodos dialógicos o discursivos presentan múltiples ventajas dentro del proceso de comprensión como forma para aproximarse a la realidad de un fenómeno objeto de investigación. De allí la aplicación de la técnica del grupo focal a los jueces. Merton y colaboradores (1990) exponen que existen varias ganancias al trabajar con entrevistas grupales, una consiste en que brinda múltiples concepciones de una misma situación, también más respuestas que pueden ser innovadoras con relación a una guía preestablecida. Adicionalmente, la intervención individual establece implícitamente una orientación para los demás, de tal forma que se generan respuestas gradualmente más personalizadas, lo cual conlleva que la discusión tome una dirección.

Con respecto al momento de la práctica discursiva, Conde (2009) acota que mientras los discursos producidos en las entrevistas individuales están centrados en el yo, en las entrevistas grupales se busca más un nosotros que puede tender a lo imaginario o a lo presente. De igual forma Fabra (2008) recuerda que para Habermas, unos de los grandes pensadores hermenéuticos, la acción social al final de cuentas es interacción, ya que es coordinación de las acciones de dos o más sujetos por medio del lenguaje. Finalmente Velásquez (2014) resalta que las relaciones sociales conforman una red de interrelaciones y su impacto se materializa en la práctica cotidiana.

\section{Procedimiento}

\section{Acceso al campo y recolección de datos}

En primer lugar estuvo el acceso al campo, definido por Rodríguez y colaboradores (1999) como un proceso por medio del cual el investigador se involucra gradualmente con el objeto de estudio. Al comienzo tiene que ver generalmente con un permiso y después se puede constituir en obtener información que solamente los par- 
ticipantes brindan a las personas en quienes confían y ocultan a los demás. Las actividades para esta parte del proyecto se dividieron en dos momentos: en primer lugar el acceso a los documentos judiciales, el cual se materializó gracias a que los investigadores se encontraban en diferentes ciudades y en cada caso se solicitó el permiso correspondiente. Con el protocolo se analizaron algunos expedientes. Esto fue útil para realizar ajustes. También se concretaron criterios de inclusión para seleccionar los documentos judiciales más pertinentes. Uno correspondía a que los documentos judiciales no fueran tan antiguos. Se acordó un intervalo de 5 años (documentos judiciales del 2010 a 2015), otro tenía que ver con el contexto sociocultural de los casos (incluir documentos judiciales de diferentes ciudades de Colombia). De la misma manera el género del juez responsable del fallo ya que se definió igual cantidad de jueces mujeres y hombres. Finalmente se eligieron los 50 expedientes.

Lo segundo fue el contacto con los jueces para la entrevista. En este caso se concretaron tres encuentros: en el primero se les comentó sobre la participación en el estudio. En otra visita se socializaron las ventajas de la investigación y los aspectos asociados al consentimiento informado $y$ compromiso de confidencialidad. Finalmente se realizó la entrevista de aproximadamente cuatro horas. En general dentro de la conversación se manejó un lenguaje sencillo y claro, de acuerdo con autores como Pava Ripoll (2015), quien enfatiza que conversar tiene un significado y utilizar un lenguaje poco elaborado posibilita que las personas se sientan partícipes de la conversación. También se contemplaron criterios sugeridos por diferentes autores, en términos genéricos, tener en cuenta la moderación del grupo, saber escuchar, utilizar el sentido del humor, manejo del ritmo de la conversación y mantener la energía vital (Callejo, 2001; Greenbaum, 1998; Krueger, 1994; Llopis Goig, 2004; Ruiz 1999). Las temáticas y preguntas guía corresponden a las mismas que orientaron en general la in- vestigación (¿los jueces toman en cuenta riesgos psicosociales para tomar las decisiones?, ¿cuáles son las causales más comunes?, ¿las causales existentes en la legislación colombiana se pueden considerar como riesgos psicosociales?, ¿se está realizando un trabajo interdisciplinario que priorice el interés superior del menor?). Los jueces se seleccionaron por medio de un muestreo intencional. Es decir, se eligieron teniendo en cuenta criterios de inclusión preestablecidos por los investigadores y estos consistían en tener más de 5 años de experiencia como jueces de familia, estar en diferentes ciudades del país y haber fallado en procesos de pérdida de patria potestad. La entrevista se grabó en audio y finalmente se transcribió literalmente para el respectivo análisis.

\section{Análisis}

Algunos investigadores expertos en análisis de expedientes judiciales (Manzanero, López, Aróztegui y El-Astal, 2015) destacan que existen limitaciones humanas a la hora de poner a jugar los procesos del pensamiento, ya que allí suelen aparecer dificultades asociadas con la capacidad de información que pueden ser consideradas, porque la realidad es multifactorial. Es así, que no se pueden tener en cuenta simultaneamente todos los aspectos. También agregan que por el contrario, una simplificación de la realidad tiene el potencial para tomar distancia de la misma. Por esta razón los investigadores analizaron por separado los expedientes. Aquí cada investigador revisó los 50 documentos preseleccionados. El protocolo facilitó centrarse en la información clave de cada documento. El siguiente paso consistió en discutir grupalmente el análisis de los documentos. En esta parte se buscaron las similitudes y diferencias de los análisis individuales. Finalmente se debatieron aspectos relacionados con la lógica y pasos o rutas que toman los jueces para llegar a la decisión final.

La entrevista se analizó por medio de una categorización inductiva. Dentro del 
ejercicio interpretativo se tomaron como referentes el soporte teórico y los objetivos del proyecto. También se identificaron categorías emergentes. De la misma manera se contrastaron similitudes y diferencias entre los participantes. Finalmente se organizó la información en cinco categorías (significados atribuidos a las causales objetivas, significados atribuidos a las causales subjetivas, significados atribuidos a otras posibles situaciones generadoras de riesgo psicosocial consideradas por los jueces, significados asignados a la relación mantenida por los progenitores y significados atribuidos al afecto en las relaciones familiares.

\section{Resultados}

\section{Documentos judiciales}

En cuanto a las situaciones asociadas a la demanda, en todos los documentos revisados se encontró que se relacionan con la competencia parental paterna. En el 100\% de los casos, las mujeres demandan la pérdida de la patria potestad al padre. Los elementos de prueba más comunes son los testimonios de familiares de mujeres demandantes, que confirman que las competencias del padre no son adecuadas, ya que generalmente se desentienden de las responsabilidades correspondientes a su rol. En algunos casos los padres se defienden argumentando que los familiares de la demandante no son idóneos para declarar. Sin embargo, se les niega dicho argumento. En cuanto a la causal más común, es el abandono por parte del padre $(90 \%)$. Dicho abandono se encuentra sustentado por la carencia de visitas y también por el atraso en las cuotas económicas pactadas. Dentro del otro $10 \%$ de sentencias se encuentra el maltrato. También en algunos casos $(20 \%)$, el padre demandado alega no ser escuchado. Sin embargo, se les aplica el art. 129 , inciso 7 del Código de Infancia y Adolescencia (2006), el cual establece que para ejercer el derecho legal sobre el niño, el padre o madre debe estar cumpliendo con las obligaciones legales respectivas (obligación de alimentar), de no estar cumpliendo, no será escuchado o escuchada.

\section{Focused group}

\section{Significados atribuidos a las causales objetivas.}

En cuanto a la causal de la privación de la libertad por un tiempo superior a un año, la mitad de los jueces manifestó estar en desacuerdo. El argumento es que existen personas privadas de la libertad que no han cometido delitos que los inhabiliten en el ejercicio de la función parental. A cuatro jueces (dos mujeres y dos hombres) les parece injusto que a un padre que se encuentra en la cárcel desde hace un tiempo y está a punto de salir, le quiten la patria potestad, ya que si ya cumplió su sentencia, no debería continuar estigmatizado e inhabilitado para cumplir sus funciones parentales. En general, expusieron un caso en el cual una mujer cuando se entera que el padre de su hijo está próximo a salir de la penitenciaría, acude inmediatamente a demandarlo. También otro caso en que una mujer dice que su abogada le recomienda no llevar a la niña a ver al padre a la cárcel porque se daña el proceso en curso. Adicionalmente, la mujer argumenta que el padre de la niña no aporta cuotas alimenticias. Por el lado de la incapacidad mental de alguno de los progenitores, consensuaron estar de acuerdo con dicha causal, pero reflexionaron en torno a un caso expuesto en la discusión. Se trata de un hombre al que se le privó de la patria potestad por su afición al juego. El argumento en este caso corresponde a que una persona que no puede administrar sus bienes personales, difícilmente podrá administrar los de otras personas.

\section{Significados atribuidos a las causales subjetivas.}

Todos los jueces concuerdan en que la causal más común es el abandono. Igualmente emerge dentro de la discusión el tema de la salida del país de los niños. Según la experiencia de los jueces, en la mayor parte de oportunidades, lo que en verdad subyace en la demanda por abandono es obtener el permiso de salida del país para el niño y las per- 
sonas demandantes buscan despojar de la patria potestad al otro progenitor para poder sacar del país al niño. Terminan concluyendo que las personas ya no quieren estar tramitando constantemente permisos de salida del país. Aclaran que el permiso de salida del país lo autoriza el progenitor que no tiene la custodia o un juez dependiendo de las condiciones en las que se pretende sacar al niño del país. Adicionalmente reflexionan los jueces en lo sospechoso que resulta que se demande a un progenitor que desde hace 10 años no visita a su hijo o tiene algún tipo de contacto. En esos casos es donde se infiere que el asunto se asocia con la salida del país del niño.

También comentan que en algunos casos el progenitor demandante busca en forma intencional que se presente un distanciamiento entre el niño y el progenitor demandado. Para ello cambian al niño de colegio, igualmente números celulares o fijos y hasta se mudan a otra residencia para disminuir el contacto. En esta parte es importante reflexionar en torno al debilitamiento del vínculo del niño con la otra figura parental, ya que distanciarlo intencionalmente puede afectar el lazo de apoyo y calidez al que tiene derecho. Esto atenta contra el interés superior del menor, debido a que según la Constitución Política Colombiana (art. 44), el derecho a tener una familia y a no ser separado de la misma, es un derecho fundamental de los niños. De igual manera el derecho a recibir cuidado y amor (por lo menos hasta los 18 años).

Significados atribuidos a otras posibles situaciones generadoras de riesgo psicosocial consideradas por los jueces

Al preguntar al grupo de jueces por otras situaciones que se pudieran considerar riesgo psicosocial como la separación prolongada de los padres, pobreza y consumo de SPA por parte de los progenitores, comentaron que generalmente no las tenían en cuenta, ya que les faltaba capacitación para poder evaluar ese tipo de problemáticas. Reconocieron que las han observado en algunos casos. También que consideran que pueden afectar el interés superior del menor. Igualmente manifestaron que debería existir más trabajo interdisciplinario entre Derecho y Psicología para mejorar este tipo de procesos.

\section{Emergentes}

\section{Significados asignados a la relación mantenida por los progenitores.}

Según las reflexiones suscitadas dentro de la discusión, es común que el progenitor que tiene la custodia y cuidado personal del niño, pida las visitas en alguna estación policial. El argumento es porque en otras épocas el progenitor que no tiene la custodia maltrataba al que la tiene. Generalmente es la madre quien solicita que las visitas se presenten de esta manera. Lo anterior evidencia un historial de relación de pareja tortuosa. El asunto es que la no resolución de conflictos correspondientes a la historia de la relación de pareja, interfiere sobre el interés superior del menor. Allí aparecen los fantasmas de infidelidades o historias de maltrato. Como consecuencia se deterioran las competencias parentales de ambos progenitores. Además, es importante tener en cuenta que el niño tendrá que asistir a los contextos policiales para encontrarse con el progenitor demandado. Igualmente, el progenitor que no tiene la custodia, puede terminar cansándose gradualmente. También considera este grupo de jueces, que el ambiente de una estación de policía no es un lugar muy idóneo para la construcción de una relación parental de calidad y calidez. Finalmente aclaran que existen casos en que es evidente que el abandono es el resultado de un desinterés del progenitor demandado, pero exponen que en realidad son pocos, ya que en la mayor parte de casos de abandono, se suelen presentan las dificultades nombradas. En cuanto al maltrato, los jueces no lo discuten mucho porque consideran que claramente es una causal que pone en peligro el interés superior del menor.

\section{Significados atribuidos al afecto en las relaciones familiares.}

El grupo de jueces concuerdan en que lo más importante es el afecto y la calidad del vínculo que se establece en las relaciones parentales. Comentan algunos ejemplos de ca- 
sos en los cuales el niño se encuentra en un hogar sustituto porque sus progenitores no les pueden garantizar una buena calidad de vida o se considera que se encuentran en riesgo psicológico y social. También exponen que con el tiempo estos niños deciden regresar. El argumento es el afecto. Igualmente resaltan que existen casos en los cuales el niño tiene todos los recursos económicos, pero la calidad y calidez del vínculo parental no es idónea. La reflexión final del focused group se concentró en la importancia del afecto y la unión familiar como mejor factor de protección para garantizar que predomine el interés superior del menor.

\section{Discusión}

En cuanto a los documentos judiciales revisados, estos no presentan mucho espacio para la reflexión en términos de la evaluación, debido a que se limitan a lógicas basadas más en una causalidad lineal progresiva o reduccionista. En la mayor parte de expedientes se observa una secuencia repetitiva que privilegia la lógica silogística. Lo anterior invita a reflexionar acerca del condicionamiento bajo el cual se encuentran los jueces a la hora de tomar decisiones, ya que parece que buscan acomodar las situaciones a la causal. Esto implicaría puntos ciegos a la hora de evaluar los hechos asociadas a la demanda. Al respecto Brooks (2002) comenta que en esta lógica existe una subordinación del juez ante la ley. Allí se utilizan una serie de operaciones lógico-mecánicas para subsumir el caso a una norma prestablecida. Para este mismo autor el formalismo de dicha operación depende del hecho de que si la norma no se presenta con claridad, el juez debería renunciar a decidir sobre la cuestión.

Los resultados del análisis de la entrevista nos invitan a reflexionar en torno a diversos aspectos. El primero es la subjetividad de los jueces a la hora de evaluar. Tanto para las causales objetivas como subjetivas es evidente que predomina la lógica realista. Según Garrido y colaboradores (2006), las decisiones menos formalistas como propuesta y enfoque para dictar sentencias en temas relacionados con la familia implican que buena parte de los fallos se basarían en las preferencias personales del juez. En este caso la experiencia con los casos tendería a ser generalizada. A lo anterior se suma la cantidad de demandas y la presión existente en torno al cumplimiento y premura del fallo, lo cual podría influir para que los encargados de impartir justicia se inclinen a tomar decisiones desde la experiencia e intuición. Para los jueces se debe revisar la causal privación de la libertad superior a un año, esto porque en algunos casos no aplicaría. El argumento tiene que ver con el hecho de que esta causal no necesariamente impide el ejercicio parental. En cuanto a la discapacidad mental argumentan que en ocasiones es muy difícil definir qué situaciones o patologías pueden en realidad afectar la competencia parental. En esta parte los jueces expusieron que no tienen suficiente capacitación en torno al tema de la salud mental. También manifiestan un gran vacío en torno al trabajo interdisciplinario. En especial en el caso de los informes, ellos comentaron que son muy técnicos y no les brindan la claridad suficiente para tomar las decisiones. En palabras de uno de los jueces: "lo que sucede es que ustedes los psicólogos y los psiquiatras, entregan unos informes con un lenguaje que no se entiende, un lenguaje para ustedes. Entonces a uno le toca descifrar jeroglíficos".

El maltrato no lo discuten, ya que es claro que atenta contra el bienestar del niño. Empero, manifiestan que es un fenómeno complejo que en muchas ocasiones pasa desapercibido y no se evalúa por falta de evidencias probatorias a diferencia de la violencia que por definición tiene que ver con el acto físico, el maltrato puede ser de diferentes tipos. Igualmente, existen personas o familias que protegen al victimario, también por una habituación o anestesia social y como consecuencia, no se denuncia. Con relación al tema, Aroca, Lorenzo y Miró (2014) indican que todavía existe desconocimiento en torno al problema. Esto se presenta porque la falta de comprensión sobre esta problemática conlleva a que algunas partes de las sociedades actuales omitan ciertas situaciones de maltrato, debido a que las entienden como normales. Adicionalmente se encuentra el papel que juega el contexto sociocultural en la construcción y mantenimiento de significados 
asociados a formas violentas de resolución de conflictos dentro de las relaciones familiares. Al respecto Barbosa González (2014) resalta que entre otros factores asociados a la violencia y maltrato familiar, se encuentra el contexto sociocultural, el cual posee un papel protagónico en la construcción, mantenimiento y transmisión intergeneracional de discursos dominantes que validan las interacciones agresivas dentro del hogar.

Por el lado del abandono y la larga ausencia, aparece una dificultad a la hora de definir estas causales. En este caso la larga ausencia del progenitor demandado puede estar asociada a una salida del país por ejemplo. Pero nuevamente entra a jugar la lógica realista del juez para determinar en qué momento la larga ausencia se puede considerar abandono. Llama la atención que en ningún expediente judicial de los revisados se observa la causal larga ausencia, en todos predomina abandono. Para este caso los jueces prefieren determinar abandono dado que larga ausencia es más ambigua y parece tener menos peso como argumento para la decisión. Esta causal es la más signifícativa dentro de los hallazgos de investigación y parece estar asociada en la mayoría de casos a buscar sacar del país al niño sin pedirle autorización al progenitor demandado. Después de analizar los documentos judiciales y la entrevista, los investigadores identificaron una secuencia o patrón que conduce a la decisión de pérdida de la patria potestad por abandono: (1) búsqueda de asesoría jurídica, (2) propiciar que la distancia física y emocional entre el progenitor demandado y el niño se torne más notoria, (3) instaurar la demanda, (4) acumular evidencia probatoria sobre el abandono (causal más frecuente) y (5) conseguir testigos, generalmente familiares, vecinos o amigos del progenitor demandante. Finalmente, el juez toma la decisión. Por supuesto que existen otras secuencias que se deberían analizar. Empero para este estudio, y teniendo en cuenta las limitaciones del mismo en cuanto a cantidad de documentos, los jueces entrevistados y los contextos socioculturales en los cuales se toman las decisiones, la secuencia más común es la expuesta con anterioridad.

Para el caso de otras situaciones generadoras de riesgo psicosocial como la separa- ción prolongada de los padres, pobreza y consumo de SPA, los jueces argumentan que generalmente las han observado. Sin embargo exponen que les falta capacitación para evaluar más situaciones que se podrían considerar dentro de las decisiones. Aquí surge una interesante reflexión en torno al tema, ya que en la separación prolongada de los padres puede aparecer un conflicto de la pareja parental sin resolver. Esta situación podría conducir a un abandono o larga ausencia de uno de los progenitores. Esto también permitiría analizar un poco más la historia de la relación de pareja y asociarla con la demanda actual. Aunque para esto el juez tendría que recurrir a una lógica circular y no a la lógica lineal progresiva del positivismo lógico.

Para los jueces la pobreza no es considerada un riego psicosocial, ya que lo importante en su opinión es el afecto y la calidad del vínculo familiar. Un juez comentó: “en mi experiencia como juez rural he podido observar padres y madres que con pocos recursos económicos mantienen relaciones de afecto excelentes con sus hijos, pero también situaciones en las que existe el dinero, pero las relaciones son de maltrato. Debido a lo anterior, he tomado la posición de que es mejor que tenga la patria potestad un padre pobre pero afectivo que un padre rico con pobres competencias parentales".

En esta parte de las entrevistas predomina la lógica realista ya porque los jueces narraban esto a partir de la experiencia con los casos. Asunto que puede constituirse en un sesgo o generalización, pero también tiene un valor importante, debido a que concuerda con los resultados de investigaciones de expertos en el tema de las relaciones parentales como Gámez, Jaureguizar, Almendros y Carrobles (2012), quienes evidenciaron que los niños que describían a sus progenitores como personas cariñosas, solían establecer vínculos menos conflictivos que los que por el contrario recordaban a sus progenitores como distantes. De la misma forma Formiga (2011) recalca que es importante que al interior de las relaciones familiares predomine un ambiente armónico, conciliador y de afecto. Finalmente, Barbosa, Segura, Garzón y Parra (2014) argumentan que la calidad y calidez de las relaciones familiares pueden funcionar 
como factor protector para la aparición de diferentes situaciones de riesgo psicosocial.

Llama la atención que no se observa la evaluación del consumo de SPA por parte de alguno de los progenitores en las sentencias y tampoco se le otorgó mayor importancia dentro de la entrevista. Esta problemática es claramente un riego psicosocial, ya que puede afectar el bienestar del niño. Al respecto Bobes (2004) comenta que cuando aparece esta problemática, se suelen observar alteraciones en el funcionamiento familiar como límites difusos y caos a nivel de las interacciones.

Otro tema adicional que se podría evaluar más a fondo en este tipo de casos son las competencias parentales. López, Casimiro, Quintana y Chaves (2009) exponen que el análisis y evaluación de las competencias parentales deberían constituirse en un tema central para quienes trabajan con servicios especializados en atención a familias que se encuentran en riesgo psicosocial, ya que en estos casos es indispensable evaluarlas para tomar medidas de apoyo o inclusive para decidir si es necesario retirar al niño de su familia. Finalmente concluyen estos investigadores que paradójicamente, precisamente esta es una de las áreas menos investigada.

\section{Consideraciones finales}

Ante la pregunta de si los jueces tienen en cuenta riesgos psicosociales para tomar las decisiones, se puede decir que lo que consideran son las causales previstas en la ley, ya que se guían a la hora de tomar la decisión por la lógica silogística, lo cual no es negativo, pero tiene el riesgo de simplificar la realidad de las situaciones evaluadas. Sin embargo, es interesante observar que dentro de la entrevista grupal utilizan la lógica realista $\mathrm{y}$ anteponen sus años de experiencia y expectativas en torno a las temáticas relacionadas con la familia. Parece que los jueces comienzan con un análisis realista de los casos. Allí pueden identificar diversos elementos de evaluación y complejizar el caso. Inclusive, es posible que logren utilizar una lógica circular o holística. Empero, en el momento de sintetizar en el documento la decisión, terminan acomodando sus evaluaciones a la causal que les exige la ley.

En cuanto a la causal más común, es interesante observar que es el abandono, pero también se encuentra que dicha causal en la mayor parte de casos, se constituye en un interés del progenitor demandante para poder sacar al niño del país sin pedirle permiso al otro progenitor. La respuesta a la pregunta acerca de si las causales existentes en la legislación colombiana se pueden considerar como riesgos psicosociales, sería afirmativa, ya que las causales efectivamente ponen en riesgo el bienestar de los niños. Sin embargo, existe falta de consenso en torno al tema. Lo que para los jueces es causal, para otros profesionales es riesgo psicosocial. Además no siempre un riego psicosocial necesariamente condicionaría el bienestar. Por ejemplo la pobreza no fue considerada por los jueces como riesgo. Es importante establecer un puente interdisciplinario para que los jueces se comuniquen con un lenguaje común con otras disciplinas que los pueden apoyar dentro de los procesos de evaluación.

Finalmente está el tema de la realización de un trabajo interdisciplinario que priorice el interés superior del menor. El trabajo interdisciplinario es muy pobre. Una prueba es que en los expedientes casi no aparece la evaluación de otros profesionales que pudieran complementar los procesos de evaluación.

Hablando de las limitaciones del estudio, los investigadores no contaron con el tiempo y recursos suficientes para realizar entrevistas grupales o individuales a más jueces en otras ciudades del país para tener unos resultados más generalizables. Igualmente, no se contó con documentos judiciales en los cuales el padre demandara a la madre. Sin embargo, el equipo de investigación logró importantes avances en torno a la comprensión de los procesos judiciales relacionados con el tema de la familia. Finalmente se resaltan los elementos interdisciplinarios del estudio, ya que tanto la Psicología como el Derecho tienen aspectos en común a la hora de evaluarsituaciones relacionadas con el desarrollo y bienestar de las personas. 


\section{Cuadro 1}

Versión abreviada del Protocolo

Proceso: Pérdida patria potestad

Demanda:

Contestación:

Se realizó durante el proceso entrevista: NO SÍ: Testigos:

Se aplicaron pruebas psicológicas: NO Sí:

Cuáles:

\begin{tabular}{|l|l|l|l|l|}
\hline \multirow{4}{*}{ CATEGORÍA } & \multicolumn{1}{|c|}{ SUBCATEGORÍA } & SI & NO & $\begin{array}{c}\text { OBSERVACIONES } \\
\text { SIGNIFICATIVAS }\end{array}$ \\
\hline \multirow{5}{*}{ Causales } & $\begin{array}{l}\text { Privación de la libertad superior a un } \\
\text { año }\end{array}$ & Maltrato & & \\
\cline { 2 - 5 } & Discapacidad del padre o madre & & & \\
\cline { 2 - 6 } & Abandono & & & \\
\cline { 2 - 6 } & Larga ausencia & & & \\
\hline \multirow{4}{*}{$\begin{array}{l}\text { Riesgos } \\
\text { psicosociales }\end{array}$} & Separación prolongada de los padres & & & \\
\cline { 2 - 6 } & Pobreza & & & \\
\cline { 2 - 6 } & Consumo de SPA & & & \\
\cline { 2 - 5 } & Otros posibles riesgos & & & \\
\hline
\end{tabular}




\section{Referencias bibliográficas}

Aroca-Montolío, C., Lorenzo-Moledo, M. \& MiróPérez, C. (2014). La violencia filio-parental: Un análisis de sus claves [Philio-parental violence: An analysis of its keys]. Anales de Psicología, 30(1), 157-170. http://dx.doi.org/10.6018/analesps.30.1.149521

Barbosa González, A. (2014). Terapia sistémica y violencia familiar: Una experiencia de investigación e intervención [Systemic therapy and family violence: A research and intervention experience]. Quaderns de Psicología, 16(2), 43-55. http://dx.doi.org/10.5565/rev/qpsicologia.1196

Barbosa, A., Segura, C., Garzón, D. \& Parra, B. (2014). Significado de la experiencia del consumo de sustancias psicoactivas en un grupo de adolescentes institucionalizados [Meaning of the experience of the use of psychoactive substances in a group of institutionalized adolescents]. Avances en Psicología Latinoamericana, 32(1), 53-69. http://doi:dx.doi.org/10.12804/apl 32.1.2014.04

Barrett, M. \& Mcintosh, M. (1995). Familia vs. sociedad [Family vs Society]. Bogotá: Tercer Mundo Editores.

Barudy, J. \& Dantagnan, M. (2010). Los buenos tratos en la infancia: Parentalidad, apego y resiliencia [Good treatment in childhood: Parenting, attachment and resilience]. Barcelona: Gedisa.

Bautista, N. (2011). Proceso de la investigación cualitativa: Epistemología, metodología y aplicaciones [Process of qualitative research: Epistemology, methodology and applications]. Bogotá: Manual Moderno.

Bobes, J. (2004). Guía de actuación preventiva para niños y jóvenes de familias con problemas de alcohol [Guide for preventive action for children and young people from families with alcohol problems]. Barcelona: Socidrogoalcohol.

Boszormenyi-Nagy, I. \& Spark, G. (2013). Lealtades invisibles. Reciprocidad en terapia familiar intergeneracional [nvisible loyalties. Reciprocity in intergenerational family therapy]. Buenos Aires: Paidós.

Brooks, R.L. (2002). Structures for judicial decisión-making from legal formalism to critical theory. Durham: Carolina Academic Press.

Bueno-Abad, J.R. (2005). El proceso de ayuda en la intervención psicosocial [The help process in psychosocial intervention]. Madrid: Popular.

Código Civil Colombiano. (2015). Ley 57 de 1887 sobre adopción y unificación de la legislación nacional. (34 ${ }^{\mathrm{a}}$. ed.). Bogotá D.C.: Legis Colección de Códigos Básicos

Callejo, J. (2001). El grupo de discusión. Introducción a una práctica de investigación [The discussion group. Introduction to a research practice]. Barcelona: Ariel.

Colombia. Asamblea Nacional Constituyente (1991). Constitución Política de Colombia. [Political Constitution of Colombia]. Recuperado el 10 de noviembre de 2017 www.banrep.gov.co/es/node/28357

Colombia. Congreso de la República. (2006). Ley No 1098 por la cual se expide el Código de la Infancia y la Adolescencia [Law No. 1098 by which the Code of Children and Adolescents is issued]. Recuperado el 10 de noviembre de 2017 de http://www.unhcr.org/refworld/docid/46d68ac12.h tml

Conde (2009). Los grupos triangulares como "espacios transicionales" para la producción discursiva: Un estudio sobre la vivienda en Huelva [The triangular groups as "transitional spaces" for discursive production: A study on housing in Huelva]. En A. Gordo \& A. Serrano (Eds.), Estrategias y prácticas cualitativas de investigación social (pp. 155-189). Madrid, España: Pearson.

Davis, C., Dearing, E., Usher, N., Trifiletti, S., Zaichenko, L., Ollen, E., Brinkoetter, M., Crowell, C., Joung, K., Parque, K., Mantzoros, C. \& Crowell J. (2014). Detailed assessments of childhood adversity enhance prediction of central obesity independent of gender, race, adult psychosocial risk and health behaviors. Metabolismo, 63(2), 199-206. http://dx.doi.org/ 10.1016/ j.metabol.2013.08.013.

Echeburúa, E. \& de Corral, P. (2009). Manual de violencia familiar [Family violence handbook]. Madrid: Siglo XXI.

Fabra, P. (2008). Habermas: Lenguaje, razón y verdad. Los fundamentos del cognitivismo en Jurgen Habermas [Habermas: Language, reason and truth. The fundamentals of cognitivism 
in jurgen Habermas]. Madrid: Marcial Pons.

Formiga, N. (2011). Valoracäo da familia e condutas desviantes: Testagem de um modelo teórico [Family valuation and deviant behavior: Testing a theoretical model]. Psico, 4 (3), 383-392.

Fuster, E.G. (2002). Las víctimas invisibles de la violencia familiar. El extraño iceberg de la violencia doméstica [The invisible victims of family violence. The strange iceberg of domestic violence]. Buenos Aires: Paidós.

Gámez-Guadix, M., Jaureguizar, J., Almendros, C. \& Carrobles, J.A. (2012). Estilos de socialización familiar y violencia de hijos a padres en población Española [Styles of family socialization and violence of children to parents in Spanish population]. Psicología Conductual, 20(3), 585-602.

Garrido, E., Masip, J. \& Herrero, C. (2006). Psicología Jurídica [Juridic Psychology]. Madrid: Pearson.

Geertz. C. (2003). La interpretación de las culturas [The interpretation of cultures]. Barcelona: Gedisa.

Gergen, K. (2007). Investigación cualitativa: Tensiones y transformaciones [Qualitative research: Tensions and transformations]. En A. Estrada \& S. Diazgranados (Eds.), Kenneth Gergen. Construccionismo social. Aportes para el debate y la práctica (pp. 245-271). Bogotá: Uniandes.

González, F. (2000). Investigación cualitativa en psicología. Rumbos y desafios [Qualitative research in psychology. Rumbos and challenges]. México: Thomson Learning.

Greenbaum, T. (1998). The handbook for focus group research. Thousand Oaks: Sage.

Kozinski, A. (1993). What $i$ ate for breakfast and other mysteries of judicial decision making, 26 loy. L.A. L. Rev. 993. Recuperado el 7 de noviembre de 2017 de http://digitalcommons. lmu.edu/llr/vol26/iss4/5

Krueger, R. (1994). Focus groups, a practical guide for applied research. London: Sage.

Llopis Goig, R. (2004). Grupos de discusión [Groups of discussion]. Madrid: Esic.

López, M., Casimiro, E., Quintana, J. \& Chaves, M. (2009). Las competencias parentales en contextos de riesgo psicosocial [Parental competences in psychosocial risk contexts]. Psychosocial Intervention, 18(2), 113-120.
Manzanero, A.L., López, B., Aróztegui, J. \& ElAstal, S. (2015). Autobiographical memories for negative and positive events in war contexts. Anuario de Psicología Jurídica, 25, 57-64. http://dx.doi.org/10.1016/j.apj.2015.02.001

Mardones, J. \& Ursúa, N. (1987). Filosofía de las ciencias humanas y sociales [Philosophy of human and social sciences]. México: Fontamara.

Masten, A. S. \& Curtis, W. J. (2000). Integrating competence and psychopathology: Pathways toward a comprehensive science of adaption in development. Development and Psychopathology, 12(3), 529-550.

Medina, J. (2008). Derecho de Familia [Family Right]. Bogotá: Universidad del Rosario.

Merton, R., Fiske, M. \& Kendal, P. (1990). The focused interview: A manual of problems and procedures. New York: Free Press.

Montenegro, M. \& Pujol, J. (2009). Derivas y actuaciones. Aproximaciones metodológicas [Derivatives and performances. Methodological approaches]. En A. Gordo \& A. Serrano (Eds.), Estrategias y prácticas cualitativas de investigación social (pp. 76-94). Madrid: Pearson.

Montoya, G. (2010). Las personas en el derecho civil [People in civil law]. Bogotá: Leyer.

Moreno, P. (2001). Concepto de marginación social [Concept of social marginalization]. En P. Moreno (Eds.), Psicología de la marginación social; concepto, ámbitos y actuaciones (pp. 67-101). Málaga: Ed. Aljibe.

Naranjo, F. (1996). Derecho civil personas y familia [Civil law people and family]. Bogotá: Librería Jurídica Sánchez.

Nardone, G., Giannotti, E. \& Rocchi, R. (2003). Modelos de familia. Conocer y resolver los problemas entre padres e hijos [Family models. Know and solve problems between parents and children]. Barcelona: Herder.

Parra, J. (2008). Derecho de Familia [Family Right]. Bogotá: Temis.

Pauli-Pott, U., Reinhardt, A., Bagus, E., Wollenberg, B., Schroer, A., Heinzel-Gutenbrunner, M. \& Becker, K. (2017). Psychosocial risk factors underlie the link between attention deficit hyperactivity symptoms and overweight at school entry. European Child \& Adolescent Psychiatry, 26(1), 67-73. http://dx.doi.org/10.1007/s00787016-0870-1 
Pava-Ripoll, N. (2015). Narrativas conversacionales con familias y docentes de niños y niñas con discapacidad: Un aporte metodológico [Conversational narratives with families and teachers of children with disabilities: A methodological contribution]. Interdisciplinaria, 32(2), 203222. https://doi.org/10.16888/interd.2012.

Rodríguez, G., Gil, J. \& García, E. (1999). Metodología de la investigación cualitativa [Methodology of qualitative research]. Málaga: Aljibe.

Ruiz, J. (1999). Metodología de la investigación cualitativa [Methodology of qualitative research]. Bilbao: Deusto.

Salgado, C. (2007). Investigación cualitativa: Metodología y retos [Qualitative research:
Methodology and challenges]. Lima: Liberabit. Serrano, R. (2007). La capacidad negocial del menor adulto. [The negotiating capacity of the adult minor]. Revista estudios socio-jurídicos. Recuperado el 7 de noviembre de 2017 de redalyc.uaemex.mx/pdf/733/73390107.pdf.

Velázquez, M. (2014). Un recorrido bibliográfico por los conceptos sujeto, sociedad, cuerpo, indumentaria y sus interrelaciones [A bibliographic tour through the concepts subject, society, body, clothing and their interrelations]. Interdisciplinaria. 31(2), 227-237.

Waters, E. \& Sroufe, L.A. (1983). Social competence as a developmental construct. Developmental Review, 3, 79-97. 